\title{
Cytotrophoblast differentiation in the first trimester of pregnancy: evidence for separate progenitors of extravillous trophoblasts and syncytiotrophoblast
}

\author{
Joanna L James, Peter R Stone and Lawrence W Chamley \\ Department of Obstetrics and Gynecology, Room 3241 C/-, Department of Surgery, Faculty of Medical and Health \\ Sciences, University of Auckland, Private Bag 92109, Auckland, New Zealand \\ Correspondence should be addressed to J L James; Email: j.james@auckland.ac.nz
}

\begin{abstract}
It is commonly accepted that a single pool of villous cytotrophoblasts are precursors of both syncytiotrophoblast and extravillous trophoblasts during the first trimester. Here we present evidence that these two trophoblast subpopulations arise from separate progenitors that have different survival characteristics when studied in villous explant cultures. Dual staining with chloromethylfluorescin diacetate and ethidium bromide revealed degeneration of the syncytiotrophoblast by non-apoptotic mechanisms within $4 \mathrm{~h}$ of culture. The syncytiotrophoblast had regenerated within $48 \mathrm{~h}$ but at this point the vast majority of the cytotrophoblast and cells of the mesenchymal core were dead. Despite this extensive cytotrophoblast death, explants are able to produce extravillous trophoblast outgrowth for up to 3 weeks in culture. We believe that the villous cytotrophoblasts in the tips of anchoring villi are resistant to the factors that cause the death of the majority of villous cytotrophoblasts in culture. We speculate that as early as $\mathbf{8}$ weeks of gestation there are two separate villous cytotrophoblast populations, one committed to differentiate into syncytiotrophoblast and the second committed to the extravillous differentiation pathway Reproduction (2005) 130 95-103
\end{abstract}

\section{Introduction}

The human placenta is a fetal organ that is essential for the development of the embryo and the success of pregnancy. The placenta acts as an interface between the mother and developing fetus, playing important roles in implantation and nutrient and gas transport, along with immune, endocrine and metabolic functions (Cross et al. 1994). In the first trimester of human pregnancy the placenta has a villous structure. Placental villi contain fetal blood vessels and occasional macrophages (Hofbauer cells) in a core of mesenchymal connective tissue, surrounded by a layer of mononuclear cytotrophoblast stem cells that is overlain by a continuous layer of multinucleated syncytiotrophoblast.

Villous cytotrophoblast stem cells differentiate along one of two possible pathways, either fusing to form the overlying syncytiotrophoblast or, in anchoring villi (those villi that physically attach the placenta to the uterus), invasive cytotrophoblasts break through the syncytiotrophoblast to form extravillous trophoblast columns (Aplin 1991). Extravillous trophoblast columns invade into the maternal decidua, physically connecting the placenta to the decidua (Pijnenborg et al. 1980, Kam et al. 1999). As the extravillous trophoblast columns move away from the anchoring villi they also spread laterally around the placenta and invade the maternal spiral arteries (Brosens et al. 1967, Kam et al. 1999). Invasion of the spiral arteries by extravillous trophoblasts leads to transformation of these vessels into large bore conduits which are necessary to allow the increased maternal blood flow that is required by the placenta/fetus as pregnancy progresses. Transformation of the spiral arteries by extravillous trophoblasts is called the 'physiological changes of pregnancy' and inadequate physiological changes of pregnancy are found in pregnancies complicated by pre-eclampsia and intra-uterine growth restriction (Brosens et al. 1967, Khong et al. 1986). Extravillous trophoblast invasion is tightly regulated both temporally and spatially, and is essential for the success of pregnancy (Cross et al. 1994, Morrish et al. 2001). The factors which drive the differentiation of villous cytotrophoblasts into either syncytiotrophoblast or extravillous trophoblast are not known. A number of interacting factors have been implicated in the migration of extravillous trophoblasts away from the placenta; however, it remains unclear which factors are essential for this process.

In this study, we have used explant cultures of first trimester human placenta to examine the behaviour of 
various trophoblast populations. Our research has led us to question the commonly held concept that a single pool of villous cytotrophoblasts are precursors of both syncytiotrophoblast and extravillous trophoblast. Rather, our results suggest that even as early as 8 weeks of gestation two separate pools of villous cytotrophoblast exist which appear to be committed to differentiation into either syncytiotrophoblast or extravillous trophoblast.

\section{Materials and Methods}

This study was approved by the regional Ethics Committee and all tissue samples were obtained with informed consent.

\section{Explant culture}

First trimester placentae were obtained following elective surgical termination of pregnancy (TOP) and washed gently in phosphate-buffered saline (PBS; pH7.4). The gestational age and fetal viability of all pregnancies prior to TOP were confirmed by ultrasound assessment. Matrigel (Becton Dickinson, Sydney, Australia) was thawed slowly at $4{ }^{\circ} \mathrm{C}$ and diluted to $1: 10$ in Dulbecco's modified Eagles' medium salts/F12 at $4{ }^{\circ} \mathrm{C}$ (DME/F12) (Life Technologies, Auckland, New Zealand). Wells of sterile 96-well culture plates (Falcon, Sydney, Australia) were coated with $50 \mu \mathrm{l} /$ well $10 \%$ Matrigel and incubated at $37^{\circ} \mathrm{C}$ for $25 \mathrm{~min}$. Excess Matrigel was removed, leaving a thin coat on each well. Villous tips were gently teased from the placentae, separated into pieces of approximately $8 \mathrm{mg}$ wet weight, and placed in the centre of each well. The villous explants were incubated at $37^{\circ} \mathrm{C}$ for $5 \mathrm{~min}$, and then $150 \mu \mathrm{l} /$ well complete trophoblast medium (DME/F12 containing $10 \%$ fetal bovine serum, $5 \mathrm{ng} / \mathrm{ml}$ epidermal growth factor, $5 \mu \mathrm{g} / \mathrm{ml}$ insulin, $10 \mu \mathrm{g} / \mathrm{ml}$ transferrin, $100 \mu \mathrm{g} / \mathrm{ml}$ streptomycin, $20 \mathrm{nM}$ sodium selenite, $400 \mathrm{U} / \mathrm{l}$ human chorionic gonadotrophin and $100 \mathrm{U} / \mathrm{l}$ penicillin) was added. The plate was then centrifuged at $210 \mathrm{~g}$ for $1 \mathrm{~min}$ in order to facilitate adhesion. The explants were cultured at $37^{\circ} \mathrm{C}$ in a humidified ambient oxygen atmosphere with $5 \% \mathrm{CO}_{2}$. Two-dimensional outgrowth of trophoblasts from the explants across the thin layer of Matrigel was observed directly by phase contrast micrography using a Nikon EL WD 0.3 phase contrast microscope with a Ph1 10 DL/0.25 Numerical Aperture (N.A.) lens (Nikon, Tokyo Japan).

\section{Assessment of cellular viability by confocal microscopy}

In total, 164 explants from 12 placentae of 8 to 12 weeks of gestation were incubated with $5 \mu \mathrm{M} 5$-chloromethylfluorescin diacetate (CMFDA) (Molecular Probes, Eugene OR, USA) in complete trophoblast medium at $37^{\circ} \mathrm{C}$ for $1.5 \mathrm{~h}$. The medium was then replaced every $10 \mathrm{~min}$ for 30 min with complete trophoblast medium only. Explants were then incubated with $2.5 \mu \mathrm{g} / \mathrm{ml}$ ethidium bromide (EtBr) (Sigma) in PBS, pH 7.4, at room temperature for $1 \mathrm{~min}$, and washed four times in PBS, pH 7.4. At each time-point, one explant was treated with Virkon (Biolab, Auckland, New Zealand) for $10 \mathrm{~min}$ before staining to serve as a control of cell death. Jeg-3 choriocarcinoma cells were used as a control to indicate cell viability. Explants were visualized in PBS at room temperature on a Leica TC SP2 confocal microscope (Leica, Oberkochen, Germany) using Leica HC PL APO $20 \times / 0.7$ N.A. Immersion Correlation Confocal Scanning and HCX PL APO $40 \times / 1.25$ N.A. Oil/Ph3 CS lenses (Leica) and photomicrographs recorded using Leica confocal software version 2.5.1104. Figures were compiled using Adobe Photoshop v5.0 (Adobe Systems, San Jose, CA, USA).

\section{Assessment of apoptosis by DNA laddering assay}

In parallel to assessment of cellular viability by confocal microscopy, at 0,48 and $96 \mathrm{~h}$ of culture 25 explants from each of the cultures established for confocal microscopy were homogenized with $500 \mu \mathrm{l}$ lysis buffer ( $4 \mathrm{M}$ guanidine thiocynate, $1 \% \mathrm{~N}$-lauryl sarcosyl and $10 \mathrm{mM}$ dithiothreitol) and incubated in a $37^{\circ} \mathrm{C}$ water bath for $30 \mathrm{~min}$ to settle. DNA was extracted from the homogenate by the method of Daniel et al. (1999). DNA was stored at $4{ }^{\circ} \mathrm{C}$, or at $-20^{\circ} \mathrm{C}$ for prolonged storage. Twenty-five microlitres of DNA extract and $3 \mu \mathrm{l}$ xylene cyanol dye $(0.25 \%$ xylene cyanol and $30 \%$ glycerol in $\mathrm{H}_{2} \mathrm{O}$ ) were loaded onto a $2 \%(\mathrm{w} / \mathrm{v})$ agarose gel and run at $100 \mathrm{~V}$. A positive control of DNA extracted by the same method from U937 cells incubated with $5 \mu \mathrm{M}$ camptothecin for $5 \mathrm{~h}$ at $37^{\circ} \mathrm{C}$ to induce apoptosis was run on every gel. Gels were stained in $0.5 \mu \mathrm{g} / \mathrm{ml} \mathrm{EtBr}$ (Sigma) in TAE buffer $(40 \mathrm{mM}$ Tris- $\mathrm{HCl}, 20 \mathrm{mM}$ glacial acetic acid and $1 \mathrm{mM}$ EDTA in $\mathrm{H}_{2} \mathrm{O}$ ) for $20 \mathrm{~min}$, rinsed in water twice for $10 \mathrm{~min}$ and visualized on a u.v. lightbox.

\section{3-(4,5-dimethylthiazol-2-yl)-2,5-diphenyltetrazolium bromide (MTT) assay}

Fresh tissue from three 8-week placentae was divided into explants which were incubated with $200 \mu \mathrm{l} /$ well complete trophoblast medium in a 96-well plate. At hourly intervals up to $5 \mathrm{~h}$ from the establishment of culture, $20 \mu \mathrm{l} 5 \mathrm{mg} / \mathrm{ml}$ MTT (Merck, Darmstadt, Germany) was added to three wells for $1 \mathrm{~h}$. Liquid nitrogen was then used to freeze MTT-treated explants in cryo-embedding medium (Biotek Scientific Supplies, Auckland, New Zealand).

The frozen tissue blocks were cut into $5 \mu \mathrm{m}$ sections using a cryostat (Leica CM1900) and collected on glass slides (BioLab Scientific, Auckland, New Zealand). Slides were fixed in $4 \%(\mathrm{w} / \mathrm{v})$ paraformaldehyde in PBS for $3 \mathrm{~min}$ and air dried for $1 \mathrm{~h}$. Slides were mounted with Aquamount (Biotek Scientific Supplies) and visualized at room temperature using Nikon plan achromat lenses of $10 \times / 0.25$ N.A. and $20 \times / 0.4$ N.A. on a Nikon Eclipse E400 light microscope. Images were captured using a 
Nikon Coolpix 990 digital camera and downloaded into Microsoft PhotoEditor v3.0.2.3 (Microsoft, Seattle, WN, USA).

\section{Immunohistochemistry}

First trimester placental tissue was divided into pieces of approximately $4 \mathrm{~mm}^{3}$ at the time of collection, or explants were removed from cultures at 4, 8, 24, 48 or $96 \mathrm{~h}$. Tissue was frozen and sectioned as described above. Slides were dipped in de-ionized $\mathrm{H}_{2} \mathrm{O}$ for $3 \mathrm{~s}$, dried for $1 \mathrm{~h}$, then fixed by immersion in cold acetone for $10 \mathrm{~min}$, air dried and stored at $-20^{\circ} \mathrm{C}$.

Non-specific binding was blocked by the addition of $100 \mu \mathrm{l} 10 \%$ normal goat serum in PBS-Tween (Life Technologies) for $10 \mathrm{~min}$ at room temperature. The slides were then washed three times with PBS, pH 7.4, containing $0.05 \%$ Tween 20 (PBS-Tween). Tissue sections approximately $25 \mu \mathrm{m}$ apart were covered with $100 \mu \mathrm{l}$ of either 1:20 M30 cytodeath antibody (Roche, Pensburg Germany) or 1:200 activated Caspase 3 antibody (Sigma) diluted in $10 \%$ normal goat serum in PBS-Tween for $1 \mathrm{~h}$ at room temperature. The slides were then washed three times with PBS-Tween and endogenous peroxidase activity was quenched by the addition of $50 \mu \mathrm{l} 5 \% \mathrm{H}_{2} \mathrm{O}_{2}$ in methanol for $5 \mathrm{~min}$. The slides were then washed three times with PBS-Tween. A Zymed Histostain-Plus kit (Zymed, San Francisco, CA, USA) containing biotinylated secondary antibody and enzyme conjugate was used according to the manufacturer's instructions. Amino ethyl carbolyl stain (Medbio, Christchurch, New Zealand) was used according to the manufacturer's instructions. Slides were then washed with de-ionized water, immersed in haematoxylin nuclear stain (Surgipath; Australian Laboratory Services, Auckland, New Zealand) for $1 \mathrm{~min}$, then washed with tap water and coverslips were mounted with Aquamount. Slides were observed at room temperature by light microscopy (Nikon Eclipse E400) as before and images captured with a Nikon Coolpix 990 digital camera. Figures were compiled using Adobe Photoshop v5.0.

\section{Explant passage cultures}

Three hundred and eighty-four explants from four placentae (two each of 9 and 11 weeks of gestation) were cultured using the methods described above (primary passage culture). On the seventh day of culture, villous explants were transplanted into a secondary passage culture by placing them into a well of a fresh Matrigel-coated plate. One hundred and fifty microlitres of fresh complete trophoblast media were added and the culture was continued for 7 days. Villous explants from the secondary passage culture were transplanted by the same method to a tertiary passage culture and the culture continued for 7 days. The outgrowth of extravillous trophoblasts from the explants in each passage was observed directly by phase contrast micrography using a Nikon EL WD 0.3 phase contrast microscope with a Nikon Ph1 10 DL/0.25 N.A. objective lens, and recorded on days 1, 2, 3, 4 and 7 of each culture using a Nikon Coolpix 990 digital camera. Prolonged cell viability in a placental explant culture of 8.2 weeks of gestation was assessed by staining with $5 \mu \mathrm{M}$ CMFDA and $2.5 \mu \mathrm{g} / \mathrm{ml} \mathrm{EtBr}$ at the end of the second passage, as described above. Tips producing extravillous trophoblast outgrowth were imaged using an inverted modified Zeiss LSM 410 confocal microscope (Zeiss, Oberkochem, Germany).

\section{Results}

\section{Viability of cell populations in first trimester villous explants}

In order to investigate the viability of trophoblasts in villous explants in culture, explants from nine placentae were cultured for up to $96 \mathrm{~h}$. Explants were stained with CMFDA, a cell-permeable dye which is metabolized in viable cells to a cell-impermeant fluorescent green dye, and $\mathrm{EtBr}$ which is only able to enter cells when membrane integrity has been compromised indicating cell death, and visualized by confocal microscopy at 4, 48 and $96 \mathrm{~h}$. After $4 \mathrm{~h}$ in culture, the earliest time-point measurable by this method, the viability of the syncytiotrophoblast layer was severely compromised as revealed by uptake of $\mathrm{EtBr}$ and failure to metabolize CMFDA (Fig. 1a). After $24 \mathrm{~h}$, fragments of dead syncytiotrophoblast were observed to be shedding from the explant (Fig. 1b). As the culture continued to $96 \mathrm{~h}$ significant areas of viable syncytiotrophoblast were evident, but the villous cytotrophoblasts underlying the syncytiotrophoblast as well as the cells of the mesenchymal core were no longer viable (Fig. 1c and d).

The MTT assay was used to assess cellular viability between 0 and $5 \mathrm{~h}$ in culture. After $1 \mathrm{~h}$ in culture all cell types in the villi were viable (Fig. 2a). The MTT staining in the villous cytotrophoblast was often more intense than that in the syncytiotrophoblast or cells of the mesenchymal core (Fig. 2a). From $4 \mathrm{~h}$ of culture, approximately half of the syncytiotrophoblast was non-viable, whereas the cytotrophoblast and cells of the mesenchymal core remained viable (Fig. 2b).

\section{Apoptosis is not the dominant cause of cell death in cultured villous explants}

In order to examine whether the loss of cellular viability in cultured villous explants was due to apoptotic death, DNA was extracted from pooled explanted villous tissue and examined for DNA laddering. DNA laddering was virtually absent from freshly harvested villous tissue, with a faint ladder present in DNA from only one of nine placentae, but DNA laddering became more frequent as the length of culture continued and was apparent in explants from six of nine placentae from $48 \mathrm{~h}$ of culture (data not shown). 

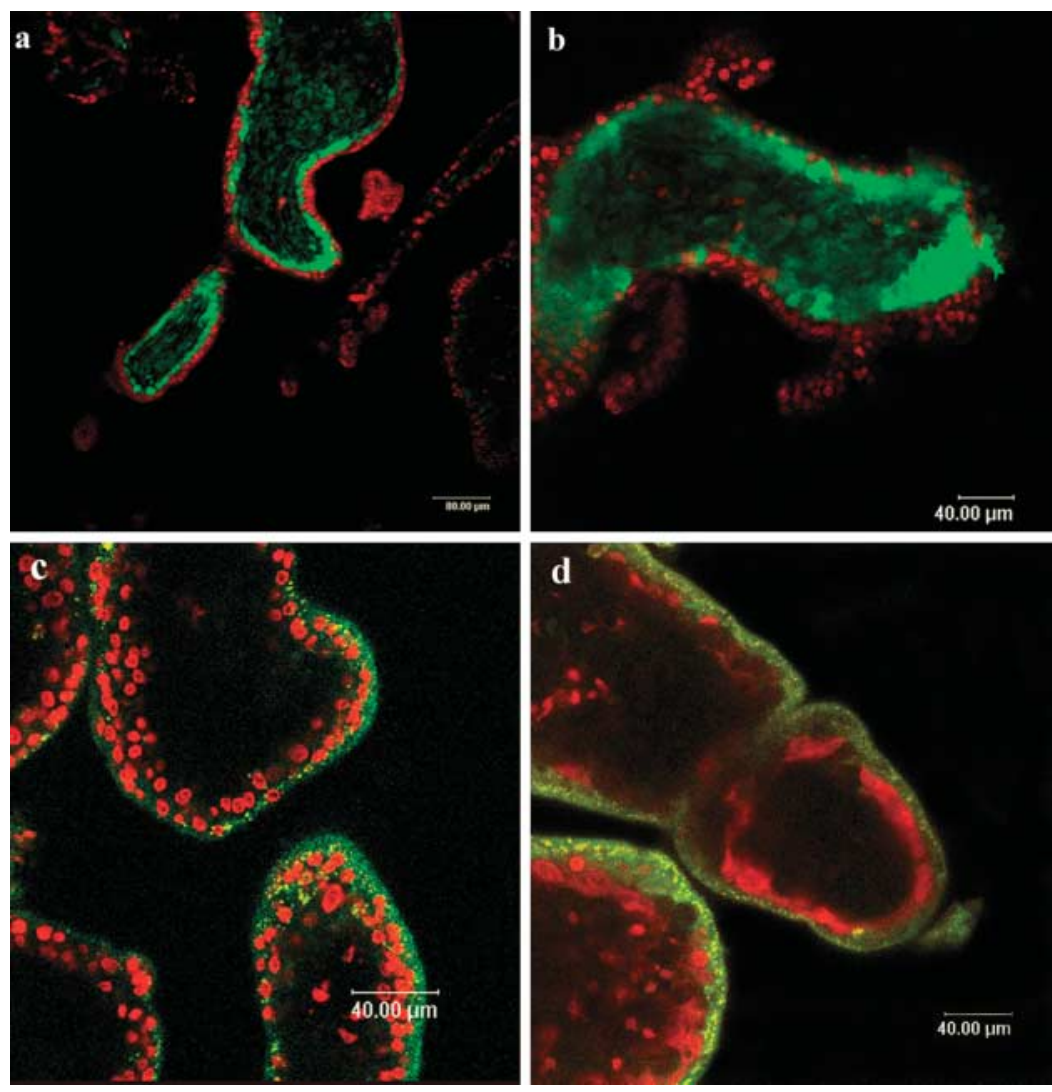

Figure 1 Optical sections of explants from a 12.2week placenta stained with $5 \mu \mathrm{M}$ CMFDA and $2.5 \mu \mathrm{M} \mathrm{EtBr}$ after (a) 4, (b) 24, (c) 48 and (d) $96 \mathrm{~h}$ of culture and visualized by confocal microscopy.

(a) After $4 \mathrm{~h}$ the syncytiotrophoblast is not viable as shown by the uptake of EtBr; however, the cytotrophoblasts and cells of the mesenchymal core metabolise CMFDA and exclude EtBr. (b) This nonviable syncytiotrophoblast layer is shed in syncytial knots as observed after $24 \mathrm{~h}$ of culture. (c) However, after $48 \mathrm{~h}$ of culture the syncytiotrophoblast has regenerated and is able to metabolise CMFDA, whereas the cytotrophoblast and cells of the mesenchymal core are no longer viable. (d) The pattern of staining seen after $48 \mathrm{~h}$ of culture is repeated after $96 \mathrm{~h}$ of culture.
In order to determine which cells in the villous explants were apoptotic, the expression of activated caspase-3 and a cytokeratin neoepitope (M30), created by cleavage of cytokeratin 18 by activated caspases, was examined by immunohistochemistry (Fig. 3). Freshly harvested villous tissue did not stain with the cytokeratin neoepitope M30 antibody and only rare villous cells contained activated caspase-3. The activated caspase-3positive cells were primarily confined to the mesenchymal core of the villi, suggesting that apoptosis was not occurring in the syncytiotrophoblast of this fresh tissue
(Fig. 3). Only small stretches of syncytiotrophoblast and occasional cytotrophoblasts stained for activated caspase-3 from $4 \mathrm{~h}$ in culture, or for the M30 cytokeratin neoepitope from $48 \mathrm{~h}$ of culture (Fig. 3). Increased expression of activated caspase-3 in the cells of the mesenchymal core was seen with extended culture, although the levels of expression in these cells varied greatly between individual explants. Although there was sporadic apoptotic death of all cell types in the explants, by and large trophoblast death appeared to be primarily non-apoptotic.

\section{A}

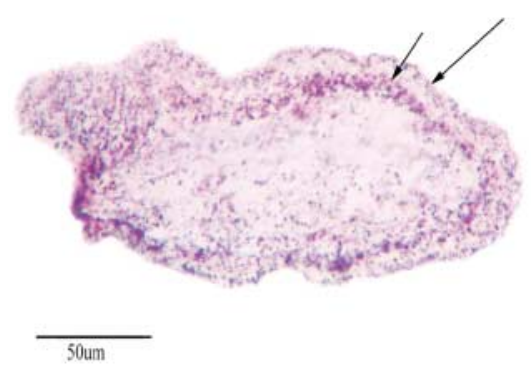

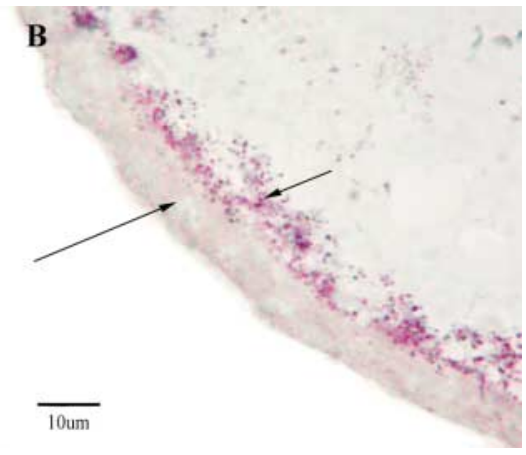

Figure 2 (a) Photomicrograph showing MTT staining of villous tissue from an explant of 8.4-weeks of gestation $1 \mathrm{~h}$ after collection. MTT staining (purple) is present in the syncytiotrophoblast, cytotrophoblast and cells of the mesenchymal core. MTT staining in the cytotrophoblast layer (short arrow) appears denser than that of the syncytiotrophoblast (long arrow). (b) Photomicrograph showing MTT staining of villous tissue from an explant of 8.4-weeks of gestation $4 \mathrm{~h}$ after collection. The syncytiotrophoblast (long arrow) is no longer viable, as shown by an absence of MTT metabolism. The cytotrophoblast (short arrow) and mesenchymal cells remain viable. 

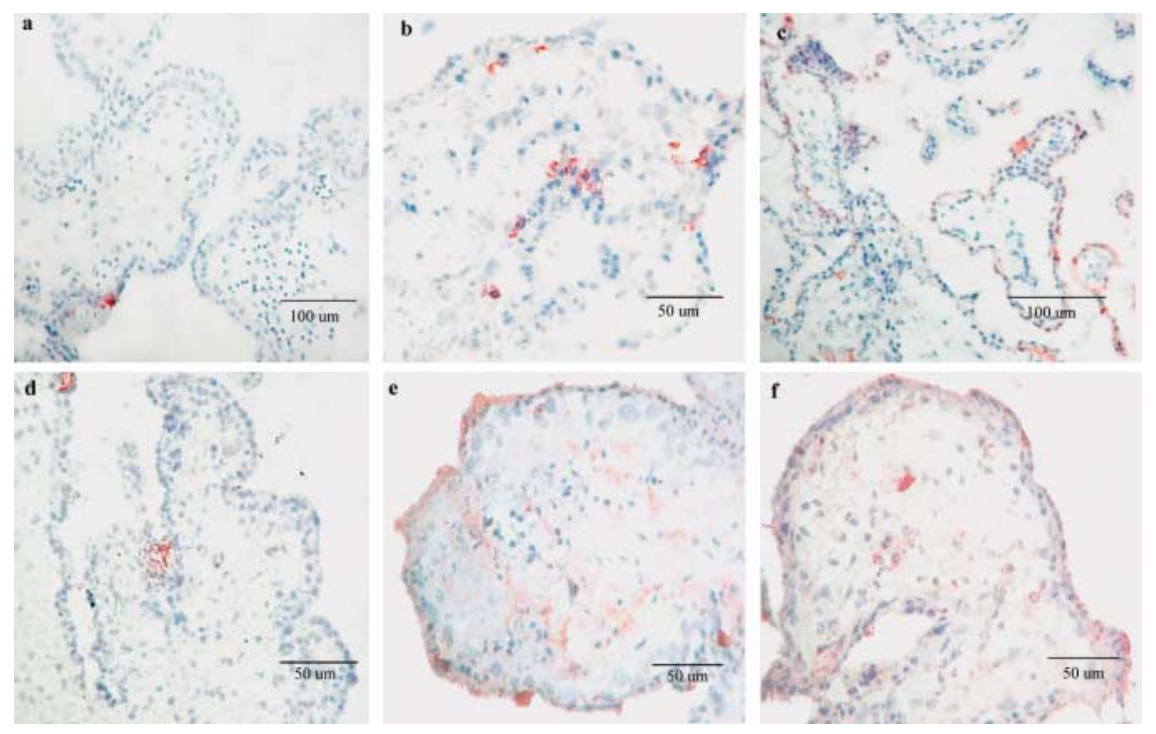

Figure 3 Photomicrographs showing immunolocalization of ( $a, b$ and c) cytokeratin neoepitope and ( $d$, e and f) activated caspase-3 in (a and d) fresh tissue and explants after (b and e) 48 and (c and f) $96 \mathrm{~h}$ of culture from an 8.5-week placenta. In fresh tissue only rare staining of isolated trophoblasts with the (a) cytokeratin 18 neoepitope-recognizing antibody M30 cytodeath or (d) activated caspase-3 was observed. However, after ( $\mathrm{b}$ and e) 48 and (c and f) $96 \mathrm{~h}$ of culture, activated caspase- 3 and the cytokeratin neoepitope M30 cytodeath stained small stretches of syncytiotrophoblast and occasional cytotrophoblasts. Activated caspase-3 staining of cells of the mesenchymal core was variable with some villi showing only occasional staining of individual cells (f), while in others mesenchymal staining was frequent.

\section{Trophoblast outgrowth continues despite the death of most villous cytotrophoblasts}

Of the explants cultured for $96 \mathrm{~h}, 19.1 \%$ produced areas of extravillous trophoblast outgrowth. A comparison of those explants that produced extravillous trophoblast outgrowth with those that did not produce outgrowth showed no difference in the viability of either the syncytiotrophoblast or villous mesenchymal core. Extravillous trophoblast outgrowth became macroscopically obvious by $96 \mathrm{~h}$ in culture despite the death of most of the villous cytotrophoblasts and cells of the villous mesenchymal core and continued to expand until it was limited by the destruction of the thin layer of Matrigel used in our culture system. At this point, the only villous cytotrophoblast population that remained viable were the pockets of multilayered cytotrophoblast that were located in villous tips directly behind the extravillous trophoblast columns (Fig. 4).

In order to examine whether extravillous trophoblast outgrowth would continue for extended periods, villous explants from four placentae were cultured for 7 days (primary culture) then transferred to fresh Matrigel-coated culture plates. The culture was continued for a further 7 days (secondary culture) and the explants were again transferred to fresh plates for an additional 7 days of culture (tertiary culture). In these experiments, 32.6, 8.0 and $3.2 \%$ of the explants produced extravillous trophoblast outgrowth in the primary, secondary and tertiary cultures respectively. Interestingly, 25\% of explants that produced trophoblast outgrowth in the second passage did not produce extravillous trophoblast outgrowth in the first passage. At the end of the second passage culture, CMFDA and $\mathrm{EtBr}$ staining confirmed that the majority of cells in the villus were not viable (Fig. 5). However, the cytotrophoblasts in the villous tips from which outgrowth was produced, and the outgrowth itself, were viable (Fig. 5).

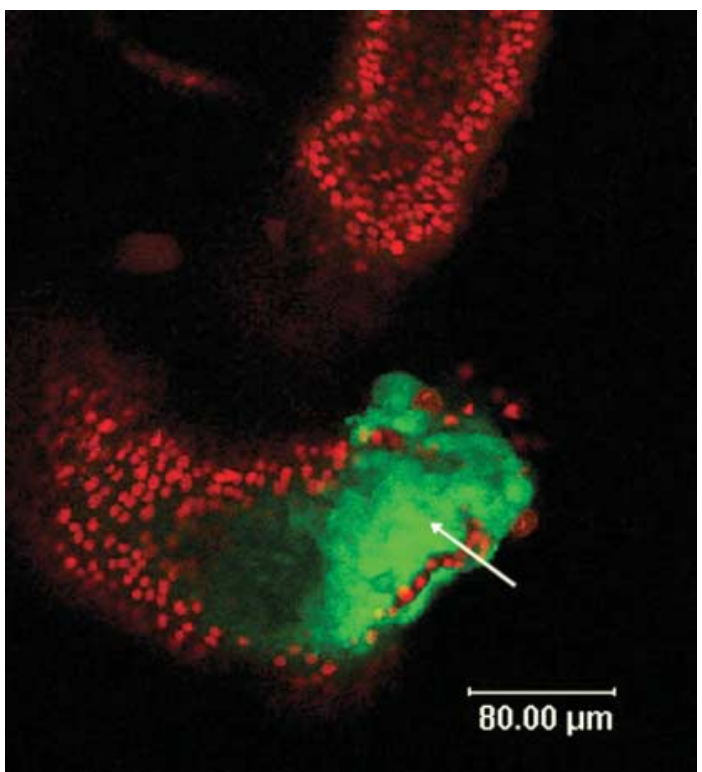

Figure 4 Optical section of a villous tip from an 8.5-week explant that produced extravillous trophoblast outgrowth, stained with $5 \mu \mathrm{M}$ CMFDA and $2.5 \mu \mathrm{M} \mathrm{EtBr}$ and visualized by confocal microscopy after $48 \mathrm{~h}$ in culture. This tip is believed to be a site of trophoblast outgrowth where the cytotrophoblasts (arrow) have remained viable despite a loss of viability in all cells of the surrounding villi. The cytotrophoblasts have breached the syncytiotrophoblast layer to produce extravillous trophoblast outgrowth of which only the first few cells can be seen in this image. 

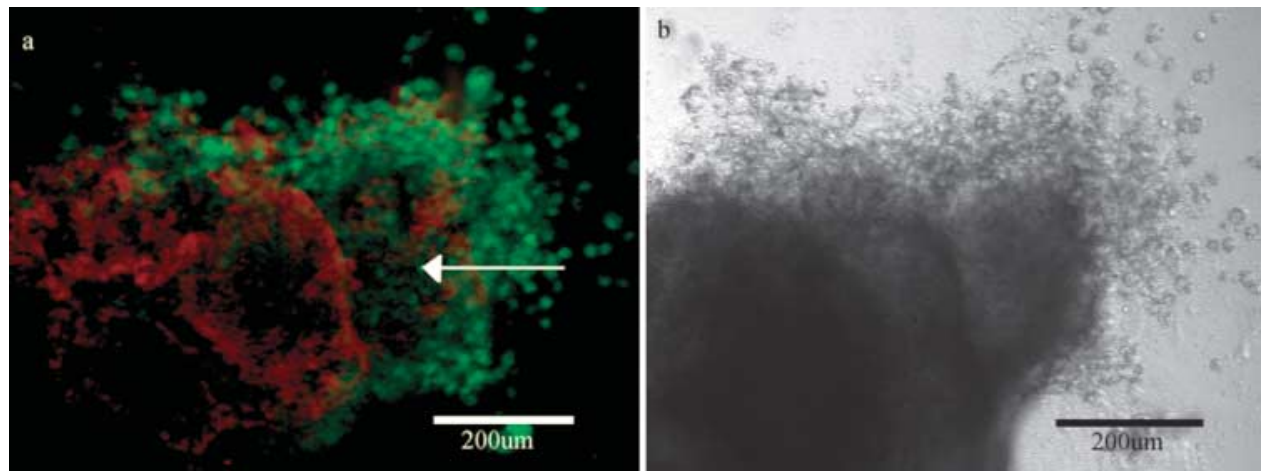

Figure 5 (a) An optical section through a villous tip from an 8.2-week placental villous explant that produced extravillous trophoblast outgrowth, and was then transferred into a fresh culture well. After one week in the second passage culture the explant was stained with $5 \mu \mathrm{M}$ CMFDA and $2.5 \mu \mathrm{M}$ EtBr. The arrow indicates viable cells in the tip from which extravillous trophoblast outgrowth was produced, whereas the majority of other cells in the villus are no longer viable. (b) Transmitted light photomicrograph of the same field shown in (a) demonstrating the morphology of the villous tip and extravillous trophoblast outgrowth.

\section{Discussion}

In order to study extravillous trophoblast differentiation, our laboratory has developed a first trimester villous explant model in which pieces of villous tissue are cultured on a very thin coating of Matrigel (an extracellular matrix substance). Unlike other models employing Matrigel, the thin coat of Matrigel means that extravillous trophoblast outgrowth in this model is two-dimensional and can therefore be readily quantified by phase-contrast microscopy and digital image analysis. Outgrowth produced from first trimester villous explants in this model was morphologically distinct and stained with antibodies against cytokeratin 7 , but not vimentin, confirming that it consists only of extravillous trophoblasts.

When establishing this model we were concerned by reports that the syncytiotrophoblast rapidly degenerates in explant cultures (Watson et al. 1995, 1998, Palmer et al. 1997, Siman et al. 2001). Palmer et al. (1997) studied cultured first trimester chorionic villi by electron microscopy, and demonstrated degeneration of the syncytiotrophoblast by $24 \mathrm{~h}$ of culture. A new syncytiotrophoblast layer was then formed from the viable underlying cytotrophoblasts by $48 \mathrm{~h}$ in culture, and was maintained until at least $120 \mathrm{~h}$ of culture (Palmer et al. 1997). We have confirmed this finding using CMFDA, an indicator of cell viability, and $\mathrm{EtBr}$, an indicator of cell death. The exclusion of $\mathrm{EtBr}$ and metabolism of CMFDA provide two independent and objective measures by which viability can be assessed simultaneously in individual cells. Using these markers, we have shown that the vast majority of the syncytiotrophoblast is non-viable after $4 \mathrm{~h}$ in culture and that large areas of the dead syncytium are shed by $24 \mathrm{~h}$ in culture. It seems likely that, despite very gentle treatment of the tissues, the initial massive syncytiotrophoblast death was triggered in the early stages of culture or during the preparation of the tissue. The syncytiotrophoblast also rapidly lost the ability to metabolize MTT after the placentae were harvested, but in contrast to the uptake of $\mathrm{EtBr}$ and lack of
CMFDA metabolism over the entire syncytiotrophoblast after $4 \mathrm{~h}$ in culture, significant areas of syncytiotrophoblast retained the ability to metabolize MTT at this time-point. However, care must be taken in using MTT as an indicator of cellular viability as the mechanism of cellular MTT reduction is not completely understood (Liu et al. 1997). MTT is generally accepted to be reduced by the mitochondrial electron transport chain, based on a study of cell homogenates by Slater et al. (1963). However, in intact cells, MTT is also able to be reduced by NADH- and $\mathrm{NAD}(\mathrm{P}) \mathrm{H}$-dependent mechanisms in intracellular vesicles, and is therefore able to be affected by factors such as oxidative stress (Liu et al. 1997). Exposure of early first trimester villous tissue, which exists in a hypoxic environment in vivo and does not contain protective antioxidant enzymes, to atmospheric oxygen concentrations would increase cellular levels of reactive oxygen species (Watson et al. 1997). High levels of reactive oxygen species have been associated with an over-reporting of cellular viability using MTT (Collier \& Pritsos 2003).

After $24 \mathrm{~h}$ in culture we observed the formation of syncytial 'knots' that are involved in the process of syncytiotrophoblast shedding from the villus. The extrusion of terminally differentiated syncytium through the formation of syncytial 'knots' is essential for the renewal of the syncytiotrophoblast and the growth of the placenta (Huppertz et al. 1998, 1999, Hempstock et al. 2003). In our cultures, the syncytiotrophoblast layer was partially regenerated as the culture progressed beyond $24 \mathrm{~h}$, whereas the underlying cytotrophoblast layer was largely non-viable. We propose that the regenerated syncytiotrophoblast was formed from the cytotrophoblasts that were viable during the first 1-2 days of culture. The syncytiotrophoblast regeneration did not completely deplete the population of underlying cytotrophoblasts, as a non-viable cytotrophoblast layer remained present at later time-points, although this layer was discontinuous in places. It is not clear why these cytotrophoblasts and the cells of the mesenchymal core died with prolonged culture, but possibly a lack of 
specific growth factors from the damaged syncytiotrophoblast or the loss of the fetal circulation contributed to the death of these cells. It is also important to note that the structure and cellular constituents of the mesenchymal core change as the villi mature and become more cellular with advancing gestation. Thus, the anchoring villi that this study concentrated on may not be entirely representative of large villi such as stem villi. Since massive cell death had occurred in these cultures the question may be asked as to why the explants remain intact. In the sterile culture system we describe there is no bacterial breakdown of the tissues. In vivo, cells of the immune system, particularly macrophages, would be responsible for removing dead cells, but in this in vitro system there are only low levels of macrophages contained in the villous core and consequently the dead but sterile tissue might be expected to remain intact for prolonged periods of time as we have observed.

The rare staining of the syncytiotrophoblast with antibodies to the cytokeratin neoepitope M30 and activated caspase-3 as well as the absence of DNA laddering that we observed suggest necrotic rather than apoptotic death of the syncytiotrophoblast, particularly during the first $24 \mathrm{~h}$ of culture. Apoptotic cell death does occur during the later stages of explant culture, but staining for activated caspase- 3 and the cytokeratin neoepitope M30 indicated that this arises from low level sporadic apoptotic death of all villous cell types and that much of the cell death that occurs during the later stages of culture is nonapoptotic. These results are in contrast to previous findings of high levels of trophoblast apoptosis within $24 \mathrm{~h}$ in term villous explants also detected using the cytokeratin neoepitope M30 antibody (Di Santo et al. 2003). However, it is possible that such differences in the level of observed apoptosis represent intrinsic differences between placentae in the first trimester and at term. It may also be possible that cell death pathways not involving the caspase pathway have been activated.

The continuation of trophoblast outgrowth from villous explants for up to 3 weeks in culture, despite the widespread death of the cytotrophoblasts underlying the syncytium, indicated that the ability of villi to produce extravillous trophoblast outgrowth and support the expansion of extravillous trophoblast columns cannot be dependent on either the cells of the mesenchymal core or the majority of the villous cytotrophoblasts, which are largely non-viable within the first week of culture. In contrast to the majority of villi, those villi from which extravillous trophoblast columns originate contain multiple layers of villous cytotrophoblasts at their tips (Vivovac et al. 1995). We have found that these multilayered villous cytotrophoblasts at the origins of trophoblast columns remain viable during prolonged culture. It is widely accepted that villous cytotrophoblasts from term placentae are committed to differentiate into syncytiotrophoblast (Morrish et al. 1997). Based on the evidence from our study we propose that as early as 8 weeks of gestation there are two distinct populations of villous cytotrophoblasts. (1) The majority of villous cytotrophoblasts form a monolayer directly beneath the syncytiotrophoblast. These cells do not survive well in the culture conditions we employed and we propose that they are committed to fusion into syncytiotrophoblast. We will refer to these as monolayer villous cytotrophoblasts. (2) Villous cytotrophoblasts that are committed to the extravillous trophoblast differentiation pathway are located in multilayered pockets at anchoring villous tips. We will refer to this type of villous cytotrophoblast as extravillous trophoblast progenitors.

The lines of evidence are that, first, although the vast majority of villous cytotrophoblasts that underlie the syncytiotrophoblast are non-viable within 4 days of culture, extravillous trophoblast outgrowth can be produced from explants for up to 3 weeks in culture. Although it is a possibility that the increased survival capacity of villous cytotrophoblast progenitors is a result of differences in the local environment of these cells, it seems unlikely that this explanation could account for prolonged survival of extravillous trophoblast progenitors for more than a few days. Whereas we have shown that extravillous trophoblast progenitors survive (as demonstrated by the production of new extravillous trophoblast outgrowth) for more than 2 weeks after the death of the cytotrophoblasts in the monolayer. It should be noted that, while new extravillous trophoblast outgrowth was generated, there was no regeneration of the syncytiotrophoblast in the region around the anchoring tips, suggesting that the cytotrophoblasts in anchoring tips do not differentiate into syncytiotrophoblast. We therefore believe that these cells are fundamentally different with an increased ability to survive in culture, allowing the formation of extravillous trophoblast outgrowth in explants when the villous monolayer cytotrophoblasts are no longer viable. The extravillous trophoblast progenitors are more resistant to the tissue preparation and culture conditions, which may reflect an important physiological adaptation by the extravillous trophoblast progenitors to equip extravillous trophoblasts for the physical environment they are exposed to in vivo when migrating from the placenta.

Secondly, this study revealed that although all explants were in contact with a permissive extracellular matrix, a significant number of explants produced extravillous trophoblast outgrowth in a secondary passage culture but not in the primary culture. It has previously been reported that contact with a permissive extracellular matrix is sufficient to stimulate trophoblast outgrowth and differentiation from explanted first trimester villi in vitro (Aplin et al. 1999). Our results suggested that the reorientation of the explants in the subsequent passage cultures brought a villous tip containing viable extravillous trophoblast progenitors into contact with the Matrigel.

Thirdly, it has been suggested that growth factors from the villous mesenchymal core, cytotrophoblast and syncytiotrophoblast play a significant role in the formation and expansion of extravillous trophoblast outgrowth 
(Bischof et al. 2000, Lacey et al. 2002). However, despite the death of the vast majority of cells in the villi within 4 days of culture, extravillous trophoblast outgrowth continued for up to 3 weeks in our model. This suggested that extravillous trophoblast progenitors are not solely dependent upon paracrine signals to drive their differentiation or invasive capacity. However, it is likely that paracrine factors would enhance trophoblast outgrowth. Alternatively, sufficient growth factors may be derived from the Matrigel which, although used as a very thin coat of diluted $(10 \%)$ Matrigel on the culture wells in this model, remains a potential source of cytokines and growth factors. The behaviour of cells in culture is dependent on the conditions of culture employed. Many workers use deep layers of concentrated Matrigel which give three-dimensional cultures. In these other models it is possible that relatively large quantities of factors that promote cell survival or other cellular behaviour are supplied by the Matrigel and thus our results may not be directly compatible with other models which use much larger amounts of Matrigel.

Other groups have previously reported phenotypic markers that distinguish the two trophoblast populations that we describe. Tenascin, an extracellular matrix glycoprotein, is present in anchoring villi immediately adjacent to sites of cytotrophoblast column initiation, but is not detected on monolayer villous cytotrophoblasts (Castellucci et al. 1992, Damsky et al. 1992). The receptor for tenascin, $\alpha \vee \beta 6$ integrin, is also expressed only by villous cytotrophoblasts at sites of extravillous trophoblast column initiation, and not in monolayer cytotrophoblasts or other villous cell types (Zhou et al. 1997). Furthermore, the binding of tenascin by $\alpha v \beta 6$ integrin stimulates cellular proliferation (Yokosaki et al. 1996). Proliferation of extravillous trophoblast progenitors would be required to drive expansion of the extravillous trophoblast columns as extravillous trophoblasts in columns do not proliferate (Vivovac et al. 1995, Korhonen \& Virtanen 1997). In contrast to extravillous trophoblast progenitors, only a small proportion of monolayer villous cytotrophoblasts stain with the proliferation marker Ki67, demonstrating another key difference between monolayer villous cytotrophoblasts and extravillous trophoblast progenitors (Vivovac et al. 1995).

Our finding that villous cytotrophoblasts from first trimester placentae do not represent bipotent progenitors could explain why it is difficult to obtain large numbers of trophoblasts that either differentiate into an invasive extravillous phenotype or proliferate following enzymatic digestion of first trimester placentae, as the vast majority of villous cytotrophoblasts are contained in the villous monolayer and we believe they are committed to the syncytiotrophoblast differentiation pathway.

The rapid degeneration of the syncytiotrophoblast in this model raises some concerns for the use of explant models in the study of syncytiotrophoblast function. In such studies, researchers should be aware of the rapid degeneration of the syncytiotrophoblast and design experiments taking this into account. However, as the observed cell death does not affect the ability of explants to produce extravillous trophoblast outgrowth, and viable trophoblast outgrowth is able to be produced for up to 3 weeks in culture, the explant model remains a good method for the study of trophoblast invasion.

In summary, in this study using a villous explant model, we have confirmed rapid death of the syncytiotrophoblast by a non-apoptotic mechanism. However, the syncytiotrophoblast is partly regenerated within $48 \mathrm{~h}$ of culture. Conversely, the majority of villous cytotrophoblasts underlying the syncytiotrophoblast and cells of the mesenchymal core die during the first week of culture. Despite this extensive villous cell death, multilayered extravillous trophoblast progenitors in the villous tips remained viable, and explants retained the capacity to produce new extravillous trophoblast outgrowth for up to 3 weeks. We believe that this demonstrates that first trimester villi contain two distinct villous cytotrophoblast populations that are committed to differentiate either into syncytiotrophoblast or extravillous trophoblast.

\section{Acknowledgements}

We wish to thank the staff at Epsom Day Unit, Greenlane Hospital, Auckland, for their help in obtaining the tissue required for this study, the patients whot donated their tissue for study and the staff of the Biomedical Imaging Research Unit, University of Auckland, for their assistance and training. This research was funded by a grant from the Evelyn Bond Trust, and J L J is a recipient of a University of Auckland Postgraduate Scholarship. The authors declare that there is no conflict of interest that would prejudice the impartiality of this scientific work.

\section{References}

Aplin JD 1991 Implantation, trophoblast differentiation and haemochorial placentation: mechanistic evidence in vivo and in vitro. Journal of Cell Science 99 681-692.

Aplin JD, Haigh T, Jones CJP, Church HJ \& Vivovac L 1999 Development of cytotrophoblast columns from explanted first-trimester human placental villi: role of fibronectin and integrin alpha5beta1. Biology of Reproduction 60 828-838.

Bischof P, Meisser A \& Campana A 2000 Paracrine and autocrine regulators of trophoblast invasion - a review. Placenta 21 Suppl A S55-S60.

Brosens I, Robertson WB \& Dixon HG 1967 The physiological response of the vessels of the placental bed to normal pregnancy. Journal of Pathology 93 569-579.

Castellucci M, Classen-Linke I, Muhlhauser J, Kaufmann P, Zardi L \& Chiquet-Ehrismann R 1992 The human placenta: a model for tenascin expression. Histochemistry 95 449-458.

Collier AC \& Pritsos CA 2003 The mitochondrial uncoupler dicumarol disrupts the MTT assay. Biochemical Pharmacology $66281-287$.

Cross JC, Werb Z \& Fisher SJ 1994 Implantation and the placenta: key pieces of the development puzzle. Science $\mathbf{1 6 6}$ 1506-1518.

Damsky CH, Fitzgerald M \& Fisher SJ 1992 Distribution patterns of extracellular matrix components and adhesion receptors are intricately modulated during first trimester cytotrophoblast 
differentiation along the invasive pathway, in vivo. Journal of Clinical Investigation 89 210-222.

Daniel PT, Sturm I, Ritschel S, Friedrich K, Dorken B, Bendzko P \& Hillebrand T 1999 Detection of genomic DNA fragmentation during apoptosis (DNA ladder) and the simultaneous isolation of RNA from low cell numbers. Annals of Biochemistry $266110-115$.

Di Santo S, Malek A, Sager R, Andres AC \& Schneider H 2003 Trophoblast viability in perfused term placental tissue and explant cultures limited to 7-24 hours. Placenta 24 882-894.

Hempstock J, Jauniaux E, Greenwold N \& Burton GJ 2003 The contribution of placental oxidative stress to early pregnancy failure. Human Pathology 34 1265-1275.

Huppertz B, Frank HG, Kingdom JCP, Reister F \& Kaufmann P 1998 Villous cytotrophoblast regulation of the syncytial apoptotic cascade in the human placenta. Histochemistry and Cell Biology $110495-508$.

Huppertz B, Frank HG, Reister F, Kingdom J, Korr H \& Kaufmann P 1999 Apoptosis cascade progresses during turnover of human trophoblast: analysis of villous cytotrophoblast and syncytial fragments in vitro. Laboratory Investigation 79 1687-1702.

Kam EPY, Gardner L, Loke YW \& King A 1999 The role of trophoblast in the physiological change in decidual spiral arteries. Human Reproduction 14 2131-2138.

Khong TY, De Wolf F, Robertson WB \& Brosens I 1986 Inadequate maternal vascular response to placentation in pregnancies complicated by pre-eclampsia and by small-for-gestational age infants. British Journal of Obstetrics and Gynaecology 93 1049-4059.

Korhonen M \& Virtanen I 1997 The distribution of laminins and fibronectins is modulated during extravillous trophoblastic cell differentiation and decidual cell response to invasion in the human placenta. Journal of Histochemistry and Cytochemistry 45 569-581.

Lacey H, Haigh T, Westwood M \& Aplin JD 2002 Mesenchymallyderived insulin-like growth factor 1 provides a paracrine stimulus for trophoblast migration. Biomed Central Developmental Biology $25-12$.

Liu Y, Peterson DA, Kimura H \& Schubert D 1997 Mechanism of cellular 3-(4,5-dimethylthiazol-2-yl)-2,5,-diphenyltetrazolium bromide (MTT) reduction. Journal of Neurochemistry 69 581-593.

Morrish DW, Dakour J, Li H, Xiao J, Miller R, Sherburne R, Berdan RC \& Guilbert LJ 1997 In vitro cultured human term cytotrophoblast: a model for normal primary epithelial cells demonstrating a spontaneous differentiation programme that requires EGF for extensive development of syncytium. Placenta 18 577-585.
Morrish DW, Dakour J \& Li H 2001 Life and death in the placenta: new peptides and genes regulating human syncytiotrophoblast and extravillous cytotrophoblast lineage formation and renewal. Current Protein and Peptide Science $\mathbf{2}$ 245-259.

Palmer ME, Watson AL \& Burton GJ 1997 Morphological analysis of degeneration and regeneration of syncytiotrophoblast in first trimester placental villi during organ culture. Human Reproduction $12379-382$.

Pijnenborg R, Dixon G, Robertson WB \& Brosens I 1980 Trophoblastic invasion of human decidua from 8 to 18 weeks of pregnancy. Placenta $13-19$.

Siman CM, Sibley CP, Jones CJ, Turner MA \& Greenwood SL 2001 The functional regeneration of syncytiotrophoblast in cultured explants of term placenta. American Journal of Physiology: Regulatory, Integrative and Comparitive Physiology 280 R1116-R1122.

Slater TF, Sawyer B \& Strauli U 1963 Studies on succinate-tetrazolium reductase system III. Points of coupling of four different tetrazolium salts. Biochimica et Biophysica Acta 77 383-393.

Vivovac L, Jones CJ \& Aplin JD 1995 Trophoblast differentiation during formation of anchoring villi in a model of the early human placenta in vitro. Placenta 16 41-56.

Watson AL, Palmer M \& Burton G 1995 Human chorionic gonadotrophin release and tissue viability in placental organ culture. Human Reproduction 10 2159-2164.

Watson AL, Palmer ME, Jauniaux E \& Burton GJ 1997 Variations in expression of copper/zinc superoxide dismutase in villous trophoblast of the human placenta with gestational age. Placenta 18 295-299.

Watson AL, Skepper JN, Jauniaux E \& Burton GJ 1998 Susceptibility of human placental syncytiotrophoblastic mitochondria to oxygenmediated damage in relation to gestational age. Journal of Clinical Endocrinology and Metabolism 83 1697-1705.

Yokosaki Y, Monis H, Chen J \& Sheppard D 1996 Differential effects of the integrins $\alpha 9 \beta 1, \alpha \vee \beta 3, \alpha \vee \beta 6$ on cell proliferative responses to tenascin. Journal of Biological Chemistry 271 24144-24150.

Zhou Y, Fisher SJ, Janatpour M, Genbacev O, Dejana E, Wheelock M \& Damsky CH 1997 Human cytotrophoblasts adopt a vascular phenotype as they differentiate: a strategy for successful endovascular invasion? Journal of Clinical Investigation 99 2139-2151.

Received 11 March 2005

First decision 14 April 2005

Accepted 22 April 2005 